\title{
CORRESPONDENCE
}

\section{Low-dose IL-2 therapy compensates for metabolic shifts and reverses anxiety-like behavior in PD-1 deficiency-induced autoimmunity}

\author{
Cui $\mathrm{Lv}^{1}$, Hao Zhang ${ }^{2,3}$, Zifa $\mathrm{Li}^{2,3}$, Hao Zhang ${ }^{1}$, Yu Zhang ${ }^{1}$, Ronghui $\mathrm{Li}^{4}$, Sheng Wei ${ }^{2,3}$ and Di Yu (D) ${ }^{1,5}$ \\ Cellular \& Molecular Immunology (2021) 18:1336-1338; https://doi.org/10.1038/s41423-020-00562-y
}

In patients with autoimmune diseases, psychological comorbidities, including anxiety, depression and cognitive dysfunction, often occur. Anxiety and depression in these patients not only cause a significant disease burden ${ }^{1}$ but also might prevent effective treatment through the neuro-immune axis. ${ }^{2}$ The latter issue results in disease aggravation and, in turn, adversely impacts psychological and social wellbeing. Therefore, effective treatment is required to break the vicious feedforward cycle of physical-psychological interactions. Although patient anxiety and depression are driven by prolonged pain and fatigue, recent studies have suggested a new mechanism whereby the dysregulated immune system can directly modulate the nervous system to induce emotional and behavioral changes. In PD-1-deficient $\left(P d c d 1^{-/}\right)$mice, a model for excessive immune activation, aberrant activation of $\mathrm{T}$ cells induces metabolic shifts by depleting amino acids, including tryptophan (Trp) and tyrosine (Tyr), which are essential precursors for monoamine neurotransmitter synthesis in the brain. Impaired brain function then results in anxiety-like behavior and exacerbates fear responses. ${ }^{3}$ In another study, $\mathrm{CD} 4^{+} \mathrm{T}$ cell-derived xanthine was shown to act on oligodendrocytes and underpin stress-induced anxiety-like behavior. ${ }^{4}$ These new discoveries suggest that anxiety and depression in autoimmune diseases can be caused, at least partially, by altered immune systems, such as excessive T-cell activation.

Recently, low-dose recombinant interleukin-2 (IL-2) treatment has emerged as a new therapy to reinstate immune balance. We and others conducted clinical trials and demonstrated the safety and efficacy of low-dose IL-2 therapy for a range of autoimmune diseases, including systemic lupus erythematosus (SLE) and vasculitis. $^{5-7}$ We also revealed that low-dose IL-2 therapy in SLE patients selectively expanded Treg cells and suppressed pro-inflammatory effector T cells. ${ }^{5}$ Here, we employed $P d c d 1^{-1-}$ mice to test whether low-dose IL-2 therapy can improve psychological alterations caused by the dysregulated immune system in autoimmune diseases.

We intraperitoneally administered low-dose IL-2 (30,000 IU every other day) in 2-month-old Pdcd1 $1^{-1-}$ or WT mice for 3 months and then examined anxiety-like behaviors of the $P d c d 1^{-1-}$ mice according to published methods ${ }^{8}$ (Fig. 1a). In the open-field test, IL-2 treatment showed no effect in WT mice but significantly improved anxiety- and locomotion-related behaviors in Pdcd1 ${ }^{-1}$ mice by partially enhancing entry into the center area and almost fully restoring the travel distance and duration in the center area to the levels observed in WT mice (Fig. 1b). In the elevated-plus maze test to assess anxiety response to open space, IL-2-treated $P d c d 1^{-1-}$ mice showed an $\sim 2$-fold increase in both open-arm entries (OEs) and open-arm staying time (OT) compared with mice receiving vehicle control (Fig. 1c). Collectively, these results suggested that low-dose IL-2 therapy could essentially reverse anxiety-like behaviors in $P d c d 1^{-1-}$ mice.

The altered behaviors of $P d c d 1^{-1-}$ mice result from metabolic shifts. ${ }^{3}$ We found that $P d c d 1^{-1-}$ mice treated with low-dose IL-2 therapy completely recovered the brain content of dopamine and 5-HT (Fig. 1d). In addition, the levels of Tyr and Trp in $P d c d 1^{-/-}$mice were almost fully restored to the levels in WT mice, not only in the brain but also in the sera (representing system amounts) (Fig. 1d). Importantly, multiple linear regression analysis demonstrated tight associations between the brain content of neurotransmitters and their amino acid precursors in both the brain and serum (Fig. 1e), indicating that low-dose IL-2 therapy in PD-1-deficient mice compensated for metabolic shifts to improve brain function.

Aberrant activation and expansion of effector T cells in Pdcd1 ${ }^{-1-}$ mice excessively consume Tyr and Trp. ${ }^{3}$ As expected, at 5-6 months of age, $P d c d 1^{-1-}$ mice, compared to WT mice, showed 2-fold and $\sim 1.5$-fold increased frequencies of CD44 ${ }^{\text {high }}$ effector $C D 4^{+}$and $C D 8^{+}$ $\mathrm{T}$ cells, respectively, while $\mathrm{CD} 62 \mathrm{~L}^{\text {high }} \mathrm{CD} 44^{\text {low }}$ naive $\mathrm{CD} 4^{+}$and $\mathrm{CD} 8^{+}$ T cells were reduced by $\sim 2$-fold and $\sim 1.5$-fold, respectively (Fig. 1f). Low-dose IL-2 therapy significantly reduced the activation and effector differentiation of CD4 ${ }^{+} \mathrm{T}$ cells in $P d c d 1^{-1-}$ mice, as shown by decreased effector cell frequencies and increased naive cell frequencies, although the protective effect was not enough to restore levels to those seen in WT mice. However, the effect of IL-2 treatment on the suppression of $\mathrm{CD}^{+} \mathrm{T}$-cell activation was milder than the effect on the suppression of $\mathrm{CD}^{+}{ }^{+} \mathrm{T}$ cells, presumably because the functional enhancement of $\mathrm{CD}^{+} \mathrm{T}$ cells by PD-1 deficiency is stronger than that of $\mathrm{CD}^{+}{ }^{+} \mathrm{T}_{\text {cells. }}{ }^{9}$

Finally, we investigated how low-dose IL-2 reduced the activation and expansion of effector $\mathrm{T}$ cells in $P d c d 1^{-1-}$ mice. We previously showed that a short period of low-dose IL-2 treatment in WT mice expanded Treg cells and enhanced their

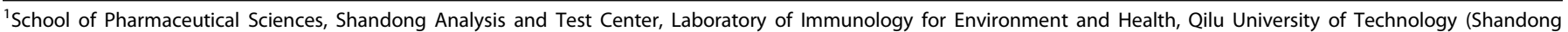

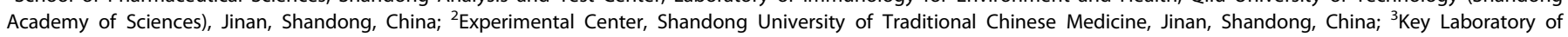

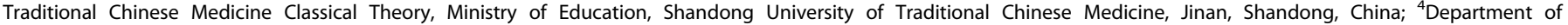

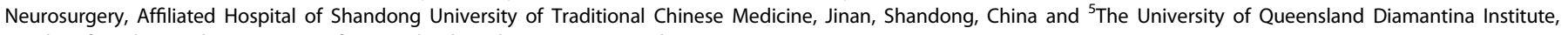
Faculty of Medicine, The University of Queensland, Brisbane, QLD, Australia

Correspondence: Sheng Wei (weisheng@sdutcm.edu.cn) or Di Yu (di.yu@uq.edu.au)

These authors contributed equally: Cui Lv, Hao Zhang
}

Received: 11 September 2020 Accepted: 17 September 2020

Published online: 16 October 2020 
Treatment with vehicle or IL-2

\begin{tabular}{|c|c|c|c|c|}
\hline \multirow{2}{*}{ WT or Pdcd1 ${ }^{-/-}$mice } & or & $\begin{array}{l}\text { ose: } 30 \text {, } \\
\text { very oth }\end{array}$ & $\begin{array}{l}\text { U) } \\
\text { y }\end{array}$ & \multirow{3}{*}{$\begin{array}{l}\text { - Open field test } \\
\text { - Elevated-plus maze test } \\
\text { - Metabolic and immunological analysis }\end{array}$} \\
\hline & 7 & 4 & 7 & \\
\hline & Month 1 & Month 2 & Month 3 & \\
\hline
\end{tabular}

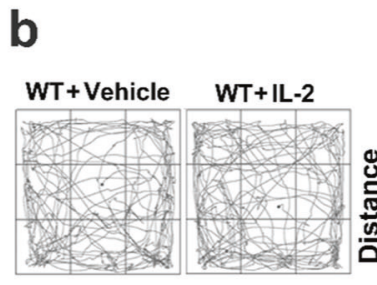

Pdcd1 $1^{-1-}+$ Vehicle Pdcd1 $1^{--}+\mathrm{IL}-2$

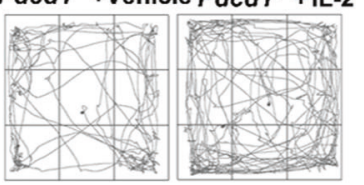

d

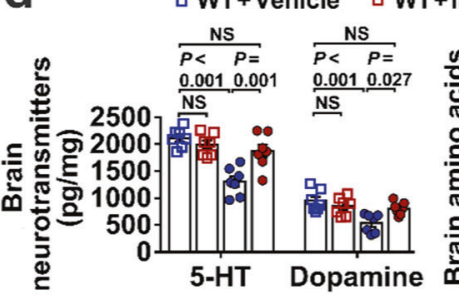

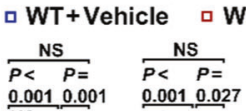
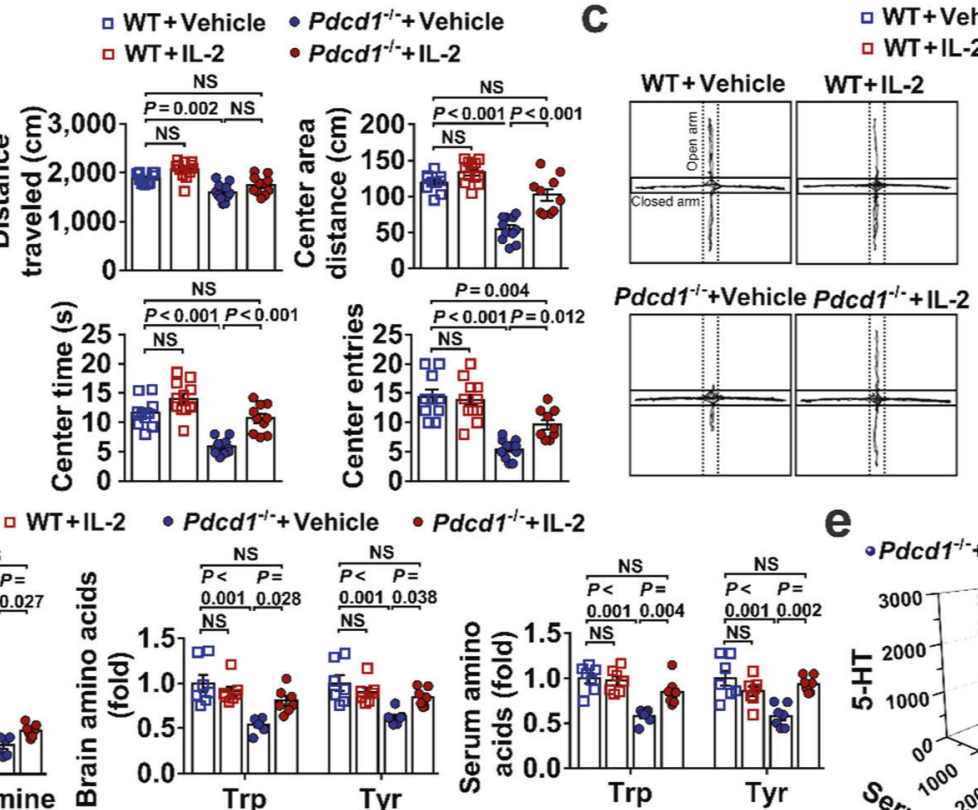

C

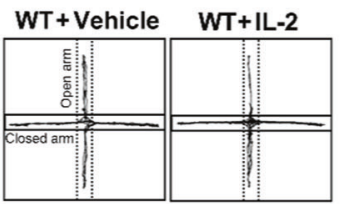

ㅁ WT + Vehicle $\bullet P d c d 1^{-i-}+$ Vehicle

a WT + IL-2 $\quad P d c d 1^{-1-}+\mathrm{IL}-2$
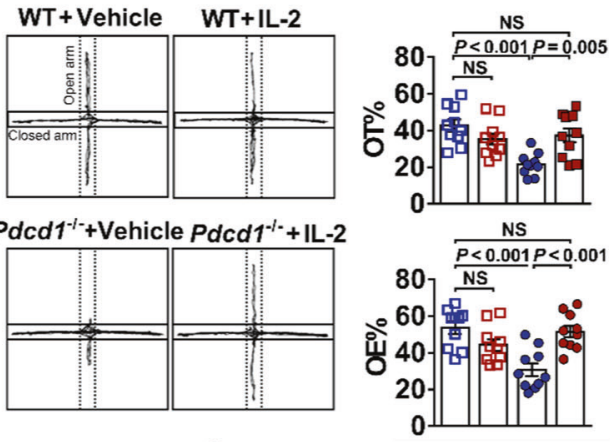

e

-Pdcd $1^{-1-}+$ Vehicle oPdcd $1^{-1-}+\mathrm{IL}-2$

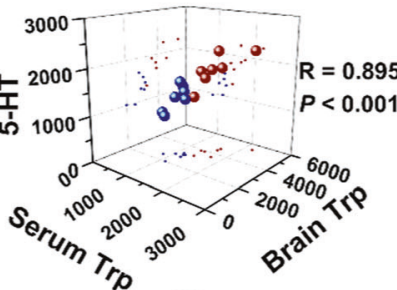

a WT+Vehicle $\bullet P d c d 1^{-1+}+$ Vehicle

$\square \mathrm{WT}+\mathrm{IL}-2$ Pdcd ${ }^{+1}+\mathrm{IL}-2$

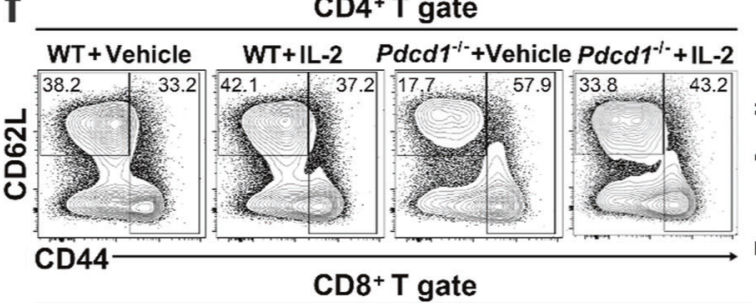

응

$80 \frac{P=0.047}{P<0.001 P=0.002} \frac{P=0.002}{P<0.001 P<0.001}$

$\frac{N S}{P<P=} \quad$ NS

0.0010 .004

응 ${ }^{1.5}$ NS

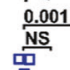

\section{NS}
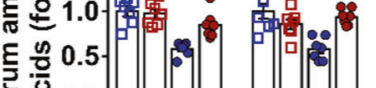

는 0.0

Trp

Tyr

¿60. NS NS

+40 啁唯

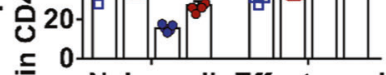

Naive cell Effector cell

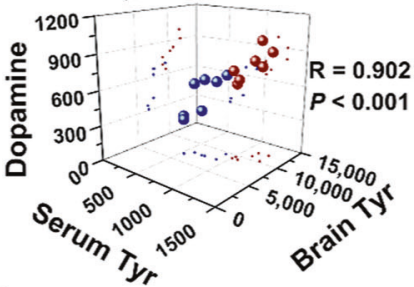

WT+Vehicle $\quad$ WT+IL-2 $\quad P d c d 1^{-1}+$ Vehicle Pdcd $1^{-1}+\mathrm{IL}-2$

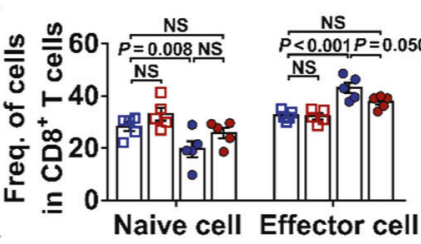

$\mathrm{h}$

Pdcd1 ${ }^{-1}+$ Vehicle Pdcd1 $1^{-1}+\mathrm{IL}-2$

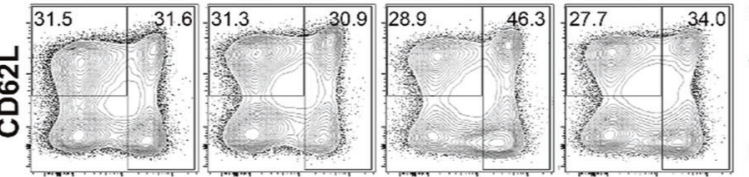

9

CD4 ${ }^{+} \mathrm{T}$ gate

$\square$ WT + Vehicle $\bullet P d c d 1^{-1}+$ Vehicle

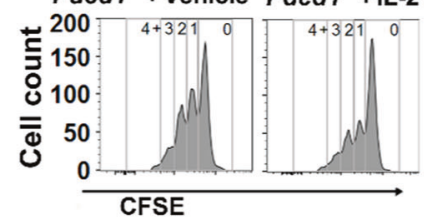

$\square \mathrm{WT}+\mathrm{IL}-2 \quad-P d c d 1^{*+}+\mathrm{IL}-2$

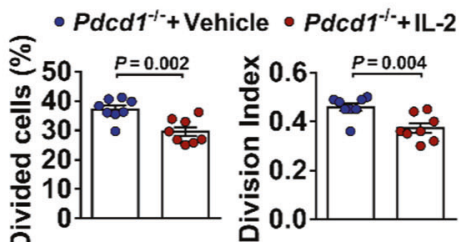

Fig. 1 Low-dose IL-2 therapy reversed anxiety-like behaviors in $P d c d 1^{-1-}$ mice by suppressing T-cell activation and compensating for metabolic shifts. a Schematic of the study design. b Results of the open-field test showing representative tracks (left) and statistics for total and center area distance traveled, entries and time spent in center areas (right). c Results of the elevated-plus maze tests showing representative tracks (left) and statistics for the percentages of time (OT\%) and entries (OE\%) in open arms (right). d HPLC-ECD analysis of the neurotransmitters dopamine and 5-HT in the brain (left), and LC-MS/MS analysis of the amino acids Trp and Tyr in the brain (middle) and serum (right). e Plots showing relationships between two independent variables (serum and brain Trp or serum and brain Tyr) with a single dependent variable (5-HT or dopamine) and the correlation result calculated as a multiple correlation coefficient ( $R$ ). $\mathbf{f}$, $\mathbf{g}$ Results of flow cytometric analysis showing representative plots (left) and statistics for CD44 ${ }^{\text {high }}$ effector and CD62L ${ }^{\text {high }} \mathrm{CD} 44^{\text {low }}$ naïve CD4 ${ }^{+}$and CD8 ${ }^{+} \mathrm{T}_{\text {cells }}$ (f) and Foxp3 ${ }^{+}$Treg cells (g) in spleens (right). $\mathbf{h}$ Results of the in vitro Treg suppression assay showing histograms for naive CD4 ${ }^{+} \mathrm{T}_{\text {-cell }}$ proliferation (upper) and statistics for the percentages of dividing cells and the division index (lower). The results are representative of three independent experiments for (b, c) $(N=10-12$ mice per group) and two independent experiments for (d-h) $(N=5-8$ mice per group). The detailed methods are described in Supplementary information 
suppressive function. ${ }^{5}$ In contrast, $P d c d 1^{-1-}$ mice that received IL2 therapy did not show expansion of Foxp $3^{+}$Treg cells (Fig. 1g), in agreement with the requirement of PD-1 for IL-2-mediated Treg expansion. ${ }^{10}$ Using widely utilized ex vivo assays to quantify the suppressive activity of Treg cells, we found that Treg cells from IL2-treated $P d c d 1^{-1-}$ mice significantly outperformed their counterparts from vehicle-treated $P d c d 1^{-1-}$ mice (Fig. $1 \mathrm{~h}$ ), suggesting that low-dose IL-2 therapy suppresses T-cell activation in Pdcd ${ }^{-1}$ mice at least partially by enhancing the function of Treg cells.

In conclusion, our study provides the first evidence that low-dose IL-2 therapy could compensate for the deprivation of essential amino acids required for neurotransmitter syntheses, protect brain function and reverse anxiety-like behaviors in PD-1-deficient mice, a model used for systemic T-cell activation and associated autoimmunity. These findings represent proof-of-concept that low-dose IL-2 as a novel therapy for autoimmune diseases can additionally benefit patients by ameliorating psychological comorbidity and improving quality of life. They also raise the possibility of combining IL-2 with other immunotherapies to offset psychological side effects.

\section{ACKNOWLEDGEMENTS}

This study was supported by the National Natural Science Foundation of China (No. 81701042 to C.L.; Nos. 81974553 and 81800187 to W.S.), the Youth Innovation Team of Shandong Provincial Department of Education (No. 2019KJK002 to W.S.) and the Taishan Scholars Program of Shandong Province and Bellberry-Viertel Senior Medical Research Fellowship (D.Y.).

\section{AUTHOR CONTRIBUTIONS}

D.Y., C.L. and W.S. designed the research; C.L., H.Z., ${ }^{2,3}$, H.Z. ${ }^{1}$ and Y.Z. performed the research; W.S., Z.F.L., H.Z. ${ }^{2,3}$, and R.H.L. contributed expertise in behavioral studies; C.L., D.Y., W.S., H.Z. ${ }^{2,3}$, H.Z. ${ }^{1}$ and Y.Z. analyzed the data; C.L. and D.Y. wrote the paper.

\section{ADDITIONAL INFORMATION}

The online version of this article (https://doi.org/10.1038/s41423-020-00562-y) contains supplementary material.

Competing interests: The authors declare no competing interests.

\section{REFERENCES}

1. Dregan, A. et al. Common mental disorders within chronic inflammatory disorders: a primary care database prospective investigation. Ann. Rheum. Dis. 78, 688-695 (2019).

2. Pavlov, V. A. \& Tracey, K. J. Neural regulation of immunity: molecular mechanisms and clinical translation. Nat. Neurosci. 20, 156-166 (2017).

3. Miyajima, M. et al. Metabolic shift induced by systemic activation of T cells in PD-1-deficient mice perturbs brain monoamines and emotional behavior. Nat. Immunol. 18, 1342-1352 (2017).

4. Fan, K. Q. et al. Stress-induced metabolic disorder in peripheral CD4(+) T cells leads to anxiety-like behavior. Cell 179, 864-879 e819 (2019).

5. He, J. et al. Low-dose interleukin-2 treatment selectively modulates CD4(+) T cell subsets in patients with systemic lupus erythematosus. Nat. Med. 22, 991-993 (2016).

6. Rosenzwajg, M. et al. Immunological and clinical effects of low-dose interleukin-2 across 11 autoimmune diseases in a single, open clinical trial. Ann. Rheum. Dis. 78, 209-217 (2019).

7. He, J. et al. Efficacy and safety of low-dose IL-2 in the treatment of systemic lupus erythematosus: a randomised, double-blind, placebo-controlled trial. Ann. Rheum. Dis. 79, 141-149 (2020).

8. Wei, S. et al. Social defeat stress before pregnancy induces depressive-like behaviours and cognitive deficits in adult male offspring: correlation with neurobiological changes. BMC Neurosci. 19, 61 (2018).

9. Konkel, J. E. et al. PD-1 signalling in CD4(+) T cells restrains their clonal expansion to an immunogenic stimulus, but is not critically required for peptide-induced tolerance. Immunology 130, 92-102 (2010).

10. Asano, T. et al. PD-1 modulates regulatory T-cell homeostasis during low-dose interleukin-2 therapy. Blood 129, 2186-2197 (2017). 